\title{
Activity-Dependent Presynaptic and Postsynaptic Structural Plasticity in the Mature Cerebellum
}

\author{
Roberta Cesa, ${ }^{1 *}$ Bibiana Scelfo, ${ }^{1 *}$ and Piergiorgio Strata ${ }^{1,2,3}$ \\ ${ }^{1}$ Department of Neuroscience and ${ }^{2}$ Istituto Nazionale di Neuroscienze, University of Turin, 10125 Torino, Italy, and ${ }^{3}$ Istituto di Ricovero e Cura a Carattere \\ Scientifico Santa Lucia Foundation, 306 Rome, Italy
}

Two models of spine formation have been proposed. Spines can derive from emerging dendritic filopodia that have encountered presynaptic partners, or presynaptic molecules may induce the spine maturation event directly from the dendritic shaft. The first model applies better to the Purkinje cell (PC), because numerous free spines have been described in several conditions, particularly when granule cells degenerate before parallel fiber $(\mathrm{PF})$ synapses are formed. A large number of new spines, many of them being free, appear in the proximal dendritic domain after blockage of electrical activity by tetrodotoxin (TTX). A complete blockage of the AMPA receptors by NBQX (2,3-dioxo-6-nitro-1,2,3,4-tetrahydrobenzoquinoxaline-7-sulfonamide), leading to a complete absence of PF- and climbing fiber (CF)evoked EPSCs and of spontaneous glutamatergic quantal events, mimics the TTX effect. In contrast, metabotropic glutamate receptor blockage by MCPG [(S)- $\alpha$-methyl-4-carboxyphenylglycine] is ineffective.

In normal conditions, in the proximal dendritic domain of the PC, clusters of a few spines are present only under each CF varicosity. It has been proposed that the active CF is responsible for spine pruning in the territory surrounding the CF synapses. Here, we show that such a pruning is mediated by AMPA but not by metabotropic receptors. Finally, after AMPA receptor blockage, there is a reduced number of spines in each spine cluster underlying CF varicosity. In conclusion, PCs tend to express spines over the entire dendritic territory. CF activity reinforces the CF synaptic contacts and actively suppresses spines in the surrounding territory, which is an effect mediated by AMPA receptors.

Key words: cerebellum; climbing fiber; neuronal activity; Purkinje cell; spinogenesis; synaptic remodeling

\section{Introduction}

Several investigations have provided evidence for different models of spinogenesis (Yuste and Bonhoeffer, 2004). According to the so-called Miller/Peters model, presynaptic terminals induce spine formation. This view is based on the demonstration that in neocortical neurons, synapses on dendritic shafts predominate at early developmental stages. In addition, in hippocampal cultures, spines can emerge in response to synaptic stimulation (Engert and Bonhoeffer, 1999). In the Sotelo model, instead, it is suggested that spinogenesis is an intrinsic property of the neuron, because in Purkinje cells (PCs), spines can develop in the absence of parallel fibers (PFs). In these two models, however, the possibility that other factors released by neuronal or non-neuronal elements are not considered. Recent in vivo imaging experiments show that spine growth also precedes synapse formation in the barrel cortex of adult mice (Knott et al., 2006). Therefore, the Sotelo model might be now extended to a more general level. A

\footnotetext{
Received June 16, 2006; revised March 13, 2007; accepted March 15, 2007.

This work was supported by grants from the Italian Ministry of University and Research, Ministry of Health, European Community contract number 512039, Regione Piemonte, Italian Space Agency, and San Paolo Foundation. We thank Dr. Shin Kang for critical comments on this manuscript and A. Renna for helpful support and technical assistance.

${ }^{*}$ R.C. and B.S. contributed equally to this work.

Correspondence should be addressed to Roberta Cesa, Department of Neuroscience, University of Turin, C.so Raffaello 30, 10125 Torino, Italy. E-mail: roberta.cesa@unito.it.

DOI:10.1523/JNEUROSCI.5617-06.2007

Copyright $\odot 2007$ Society for Neuroscience $\quad$ 0270-6474/07/274603-09\$15.00/0
}

first open issue, however, is whether spine formation requires glutamate released in the extracellular environment by different structures including non-neuronal cells such as glia (Fellin et al., 2005).

In the cerebellar cortex, a high density of spines innervated by the PFs characterizes the distal dendritic domain of the PC. In contrast, in the proximal domain, clusters of a few spines are present under each of the near 300 varicosities of the climbing fiber (CF) terminal arbor (Cesa and Strata, 2005). After a block of the electrical activity for $7 \mathrm{~d}$ in vivo, a large number of new spines emerge from the PC proximal dendritic domain, and the new spines are innervated by the PFs (Bravin et al., 1999; Morando et al., 2001). Because lesion of the inferior olive, the source of CFs, results in a similar hyperspiny transformation, CF activity is assumed to suppress spinogenesis in the region surrounding the synaptic site. Therefore, control of spinogenesis should not be seen as a simple interaction between an intrinsic property of a neuron and a spine-inducing mechanism by an excitatory presynaptic partner but rather as a result of different interacting mechanisms, including active repression.

As a second issue, we aimed at finding out which types of glutamate receptors mediate the local repressive action on spinogenesis. Adult cerebellar PCs express different subunits of the ionotropic AMPA/kainate (Lambolez et al., 1992; Petralia and Wenthold, 1992) and metabotropic (Nusser et al., 1994; Petralia et al., 1998) glutamate receptors (mGluRs). Therefore, to address 
these issues, we infused selective antagonists of these receptors for $7 \mathrm{~d}$ in vivo in cerebellar parenchyma of adult rats. We used 2,3dioxo-6-nitro-1,2,3,4-tetrahydrobenzoquinoxaline-7-

sulfonamide (NBQX) to block AMPA/kainate receptors and $(S)$ $\alpha$-methyl-4-carboxyphenylglycine (MCPG) to block mGluRs. Using this procedure, glutamate released either by the presynaptic structure as well as that released by non-neuronal elements is prevented to exert its action on the postsynaptic sites. Finally, because CFs and PCs show remarkable reciprocal interactions required for the maintenance of the correct synaptic connectivity (Cesa and Strata, 2005), we analyzed the morphological features of CF-PC synapses after the receptor block.

\section{Materials and Methods}

Experimental animals. The study was performed on adult Wistar albino rats (body weight, 150-250 g; age, 1.5-3 months; Charles River, Calco, Italy). All surgical procedures were performed under general anesthesia by a mixture of ketamine $(100 \mathrm{mg} / \mathrm{kg}$ Ketavet; Gellini Farmaceutici, Latina, Italy) and xylazine (5 mg/kg Rompum; Bayer, Leverkusen, Germany). The experimental plan was designed in accordance with the Council Directive of November 24, 1986 (86/609/EEC) of the European Community, the National Institutes of Health guidelines, and the Italian law for care and use of experimental animals (DL116/92) and was approved by the Italian Ministry of Health and the Bioethical Committee of the University of Turin.

Pharmacological treatment. To block AMPA receptors and mGluRs in vivo, we chronically infused for $7 \mathrm{~d}$ NBQX or MCPG (Tocris Cookson, Bristol, UK) into the dorsal vermal cortex by means of an osmotic minipump (Alzet 2002; Alza, Cupertino, CA). To assess the dose sufficient to completely block postsynaptic AMPA receptors, we infused 1, 1.5 , and $2 \mu \mathrm{mol}$ of NBQX in $100 \mu \mathrm{l}$ of physiological solution for each rat (see below). For MCPG, we used the maximal concentration achievable with this drug: $1 \mu \mathrm{mol}$ in $100 \mu \mathrm{l}$ of physiological solution for each rat (Tocris Cookson). Control rats were infused only with physiological solution.

Slice preparation and electrophysiology. Cerebellar parasagittal slices (200 $\mu \mathrm{m}$ thick) for electrophysiological recordings were prepared according to previously described protocols (Llinàs and Sugimori, 1980; Edwards et al., 1989). The rats were anesthetized with halothane (Fluothane; Zeneca, London, UK) and decapitated. The cerebellar vermis was rapidly removed and placed in an ice-cold extracellular medium containing the following (in mM): $125 \mathrm{NaCl}, 2.5 \mathrm{KCl}, 2 \mathrm{CaCl}_{2}, 1 \mathrm{MgCl}_{2}$, $1.25 \mathrm{NaH}_{2} \mathrm{PO}_{4}, 26 \mathrm{NaHCO}_{3}$, and 20 glucose and was bubbled with $95 \%$ $\mathrm{O}_{2} / 5 \% \mathrm{CO}_{2}$ so that the $\mathrm{pH}$ was 7.4 . Slices were cut using a vibratome (Vibroslice 752; Campden Instruments, Sileby, UK) and kept for the first hour at $35^{\circ} \mathrm{C}$ and then at $25^{\circ} \mathrm{C}$ for the rest of the day. After $1 \mathrm{~h}$ of incubation, one slice at a time was transferred to the recording chamber perfused with extracellular medium at room temperature. Identification of PCs was achieved on slices when viewed with a $40 \times$ water-immersion objective mounted to an upright microscope (BX51WI; Olympus Optical, Tokyo, Japan) (Edwards et al., 1989; Konnerth et al., 1990). All experiments were performed in whole cell with borosilicate pipettes (Hilgenberg, Malsfeld, Germany). Their resistance was 2-3 $M \Omega$ when filled with the intracellular solution containing the following (in mM): 137 K-gluconate, $2 \mathrm{MgCl}_{2}$, $10 \mathrm{HEPES}, 4 \mathrm{Na}$-ATP, $0.4 \mathrm{Na}$-GTP, and 10 EGTA, $\mathrm{pH} 7.3$, adjusted with $\mathrm{KOH}$. In a subset of experiments, in which we also tested the GABAergic responses, we used an intracellular solution composed of the following (in $\mathrm{mm}$ ): $120 \mathrm{CsCl}, 20$ tetraethylammonium, 10 EGTA, 10 HEPES, $4 \mathrm{MgCl}_{2}$, $4 \mathrm{Na}$-ATP, 0.4 GTP sodium salt, $\mathrm{pH}$ 7.3, adjusted with $\mathrm{CsOH}$. In these recording conditions, the reversal potential for chloride was $-2.58 \mathrm{mV}$. At the holding voltage of $-65 \mathrm{mV}$, positive inward currents were recorded as the result of GABA receptor-mediated outflow of chloride ions (see Fig. 2).

Ionic currents were recorded with an EPC8 amplifier (HEKA, Lambrecht, Germany). The traces were filtered at $3 \mathrm{kHz}$ and digitized at 20 $\mathrm{kHz}$. Synaptic responses were recorded in PCs from the same slice either close to the site of infusion at a distance of $\geq 200 \mu \mathrm{m}$ (near site) and at a distance of approximately three lobules from the same site (far site) as a control. For stimulation of CFs, one sodalime glass pipette (Hilgenberg), 5-10 $\mu \mathrm{m}$ in diameter filled with standard saline, was placed in the granular layer $\sim 100 \mu \mathrm{m}$ away from the recorded PC. Negative current pulses (duration, $100 \mu \mathrm{s}$; amplitude, $0-150 \mu \mathrm{A}$ ) were applied for focal stimulation, and CF responses were identified by their all-or-none feature and paired-pulse depression (Konnerth et al., 1990; Perkel et al., 1990).

Responses to PF stimulation were recorded in the same cell by placing the stimulation pipette in the molecular layer above the recorded PC. Current pulses were delivered with increasing amplitude from $0 \mu \mathrm{A}$ with steps of $5 \mu \mathrm{A}$. The identification of the PF-EPSC was based on the graded development to smoothly increasing stimulus intensity and by the paired-pulse facilitation. mGluR activation was induced in the presence of NBQX $(20 \mu \mathrm{M})$ in the external medium with a short high-frequency train of pulses to the PFs (10-15 pulses at $100 \mathrm{~Hz}$ ) (Batchelor et al., 1994; Tempia et al., 1998).

Histological procedures. At the end of the survival period, under general anesthesia, rats were transcardially perfused with $500 \mathrm{ml}$ of paraformaldehyde (4\%) in $0.12 \mathrm{~m}$ sodium phosphate buffer ( $\mathrm{PB}, \mathrm{pH} 7.4)$. The brains were dissected, kept in the fixative overnight at $4^{\circ} \mathrm{C}$, cryoprotected, and cut in several series of $30-\mu \mathrm{m}$-thick sagittal sections using a cryostat. We used a calbindin antibody to label PCs and an antibody raised against the vesicular glutamate transporter 2 (VGlut2) to visualize CF boutons (Fremeau et al., 2001; Ichikawa et al., 2002; Miyazaki et al., 2003).

One series of sections was incubated overnight with a mixture of mouse monoclonal calbindin D-28K antibody (1:2000; Swant, Bellinzona, Switzerland) and rabbit VGlut2 antibody (1:500; SYSY, Göttingen, Germany). The following day, sections were treated for $1 \mathrm{~h}$ with the following secondary antibodies: horse anti-mouse coupled to fluorescein isothiocyanate (1:200; Millipore, Bedford, MA), and biotinylated goat anti-rabbit (1:200; Vector Laboratories, Burlingame, CA) with Texas Red avidin (1:200; Vector Laboratories). The reacted sections were mounted on chrome alum gelatinized slides and coverslipped.

Confocal imaging of calbindin-labeled PC dendritic spines and VGlut2labeled varicosities. Immunostained sections were used to obtain images with a Fluoview confocal laser-scanning microscope (Olympus Optical). Three rats from each experimental group were used for quantitative analysis. For each cerebellum, we randomly acquired five images of the molecular layer with at least one segment of PC proximal dendrite. Proximal and distal dendrites were discerned according to the two different calibers, and only those having a diameter above and below $2 \mu \mathrm{m}$, respectively, were retained for quantitative analysis (Bravin et al., 1999). A $100 \times$ oil-immersion lens and an additional electronic zoom factor of $1.5 \times$, to clearly resolve dendritic spines, were used. We collected a variable number of optical section images in the $z$-dimension ( $z$-spacing, 0.5 $\mu \mathrm{m}$ ) ensuring that segments of proximal dendrites, spanning multiple confocal planes, were fully captured. The lengths of dendrite segments used for the spine quantitative analysis ranged between 10 and $30 \mu \mathrm{m}$ each. We used the same images to measure the spine density in segments of distal dendrites ranging between 2 and $13 \mu \mathrm{m}$ length.

For the spine density evaluation, individual images from the stack were printed. The spine density evaluation was calculated by collecting only the spines emerging from the proximal and distal dendrite in the central image of the series. Therefore, the quantitative spine evaluation was underestimated if compared with the total number of the spines emerging from the entire dendrite but suitable for detecting density difference among the experimental groups. Each spine emerging from the proximal dendrite, identified in a given section, was followed until it disappeared downstream and upstream in the image series in order not to include the sample spines emerging from other dendritic segments. The lengths of sampled proximal and distal dendrites were measured by means of Scion (Frederick, MD) Image software. The spine density was expressed as the number of spines per micrometer of dendrite length.

For the evaluation of the CF terminal arbor, the same optical section images were projected into a single one: all labeled varicosities distributed along the proximal dendrites were counted. The lengths of sampled proximal dendrites and varicosities were measured by means of Scion Image software to calculate the number of labeled varicosities per micrometer of dendrite membrane length, major axis, and minor axis of each varicosity. 
The evaluation of the density of CF varicosities per area was performed in different images (single optical section) of the entire molecular layer, acquired with a $40 \times$ oil-immersion lens with an additional electronic zoom factor of $1.5 \times$. The areas were measured by means of Scion Image software and the density expressed as number of varicosities per square micrometer of molecular layer. The same images were used to analyze the percentage of extension of the CF varicosities in the molecular layer. Statistical evaluations were performed by using a Student's $t$ test.

Electron microscopy. Ultrastructural analysis was performed on vehicle- and NBQX-infused rats killed after $7 \mathrm{~d}$ of treatment $(n=3$ for each group). Under general anesthesia, rats were transcardially perfused with $1000 \mathrm{ml}$ of Karnovsky double aldehyde fixative solution (1\% paraformaldehyde $/ 1 \%$ glutaraldehyde in $0.12 \mathrm{M} \mathrm{PB})$. The brains were dissected and kept in the same fixative at $4^{\circ} \mathrm{C}$ overnight. The cerebellar vermis was cut in 1-mm-thick sagittal slices by a vibrating blade microtome (Leika, Wien, Austria) and further reduced into small tissue blocks. Cerebellar blocks were postfixed in a $2 \%$ osmium tetroxide solution, dehydrated, and embedded in Epon/Araldite resin (Fluka, NeuUlm, Germany; or Sigma, St. Louis, MO). After contrast enhancement with uranyl acetate and lead citrate, ultrathin sections $(90-110 \mathrm{~nm})$ were observed on a JEM-1010 electron microscope (Jeol, Tokyo, Japan) equipped with a side-mounted CCD camera (Mega View III; Soft Imaging System, Munster, Germany). The presynaptic terminals were identified by their morphology: small clusters of vesicles abutting the synaptic contact characterize PF terminals. In contrast, CF boutons contain a high density of vesicles and some dense core vesicles. These excitatory boutons, containing round vesicles, contact the PC dendrite onto the spines forming asymmetric type synapses. A third type of input to the PC is made by the terminals of inhibitory interneurons; they are characterized by round and oval or flattened vesicles and form symmetric-type synapses onto the smooth surface of dendrites (Palay and Chan-Palay, 1974). Morphological CF varicosity measurements were performed by means of analySIS Docu software (Soft Imaging System, Munster, Germany), which delineates the axis and the contour of the acquired terminals, and then it calculates lengths, perimeters, and areas of the selected frames. The Student's $t$ test was used for statistical analysis.

\section{Results}

Electrophysiological evidence of glutamatergic receptor block After the administration of NBQX during a period of $7 \mathrm{~d}$, we have recorded, in the cerebellar slices, the postsynaptic currents evoked in PCs by PF and CF stimulation. In the rats whose cerebella were perfused with 1 and $1.5 \mu \mathrm{mol}$ of NBQX, we found that PF- and/or CF- evoked EPSCs were present respectively in three of four and in five of seven cells recorded near the site of infusion. On the contrary, in those rats whose cerebella were infused with 2 $\mu \mathrm{mol}$, AMPA-mediated responses near the site of infusion were completely abolished up to $2 \mathrm{~h}$ from slice preparation $(n=22)$. Furthermore, in these experiments, to prove that possible effects resulting from the long-lasting action of the blocker did not abolish presynaptic release, we assessed the presence of mGluRmediated response with a short high-frequency train of pulses to the PFs. In the first group of rats, immunohistochemical analysis showed no changes in spine profile in the PC proximal dendrites. In contrast, in rats receiving $2 \mu \mathrm{mol}$, we observed a remarkable increase (see below).

Concerning MCPG, we found that $1 \mu \mathrm{mol}$ infused during a period of 1 week completely blocked the postsynaptic mGluRmediated postsynaptic current without affecting the spine profile of the proximal dendritic domain. On the basis of this finding, further experiments have been performed by using $2 \mu \mathrm{mol}$ of NBQX and $1 \mu \mathrm{mol}$ of MPCG. Similar to the NBQX experiments, before recording the mGluR current in the near site, we checked for AMPAR-mediated responses to ensure that lack of mGluR current was because of the infusion of the antagonist and not because of effects other than specific block of the receptors.
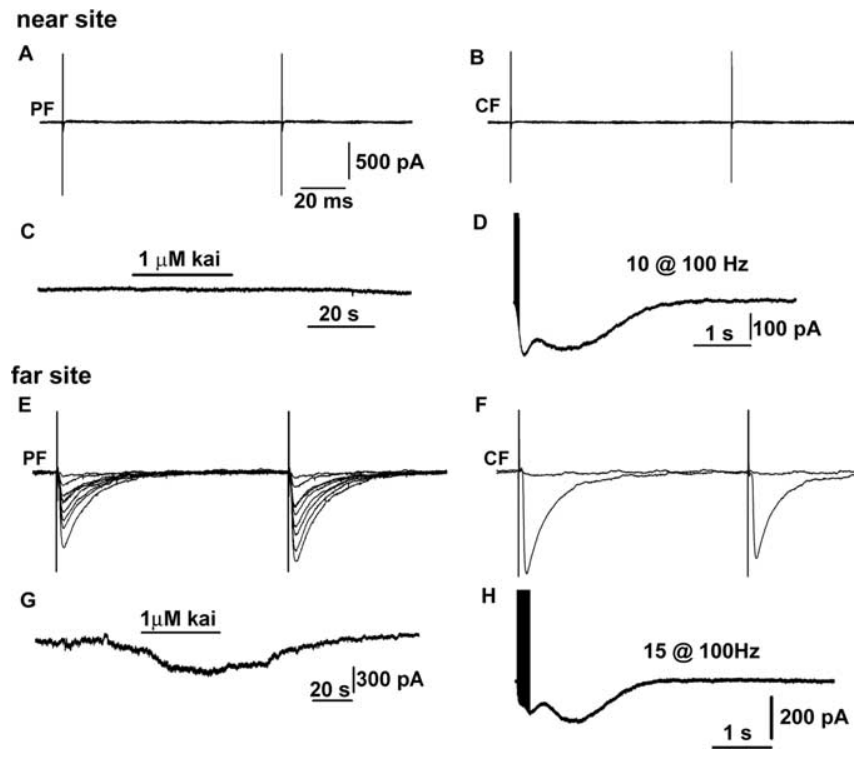

Figure 1. Assessment of EPSCs in PCs from treated cerebella. $\boldsymbol{A}, \boldsymbol{B}$, Stimulation in the molecular layer at gradually increasing strengths at the near site of an NBQX-treated cerebellum induces no PF- $(\boldsymbol{A})$ or CF-EPSC $(\boldsymbol{B})$ in the recorded PC. In the far site of the same slice, PF stimulation evokes the typical graded responses $(\boldsymbol{E})$, and $(F$ stimulation elicits the typical allor-none response $(\boldsymbol{F}) . \boldsymbol{D}, \boldsymbol{H}$, EPSC recorded in the near and far sites, respectively, by highfrequency stimulation of the PFs in NBQX-treated rats. Kainate (kai) application in the near site (C) evokes no response in NBQX-treated animals, whereas an inward current is present in the far site $(\boldsymbol{G})$.

In each slice, recording was made both in the neighborhood of the infusion site (near site, distance between 200 and $400 \mu \mathrm{m}$ ) and at a distance of at least three lobules sideways (far site, $\sim 2-4$ $\mathrm{mm}$ ) to be considered as control. The slices were kept in a recovering chamber for a minimum of $45 \mathrm{~min}$ after the cut and then placed in a dish for immediate recording. In the NBQX-treated rats $(n=9)$, within a $2 \mathrm{~h}$ period, we recorded a total of $30 \mathrm{PCs}$ near the infusion site and 21 cells located in the far site. In none of the cells recorded near the injection site, we found a trace of PFand CF-evoked EPSCs (Fig. 1 $A, B$ ). In eight cells recorded after $2 \mathrm{~h}$ from the section, we found a CF-EPSC of reduced amplitude $(<20 \%$ of the CF amplitude in the near site). In contrast, we recorded PF- and CF-EPSCs in 19 of 21 PCs located at the far site (Fig. 1E,F).

It is possible that the absence of responses to PF and CF stimulation after chronic infusion of NBQX is because of secondary effects on presynaptic terminals such as changes in retrograde signaling from PCs affecting glutamate release (Kakizawa et al., 2005). These authors, nevertheless, found a decrease, but not a complete suppression of glutamate release. However, to rule out the possibility that in our experimental conditions the lack of AMPA-mediated responses is because of a complete suppression of such a release, we assessed the presence of mGluR-mediated EPSC in the near and far sites of NBQX-infused animals. In 6 of 16 cells tested at the near site, we were able to elicit mGluR response in the absence of AMPA-mediated current after PF stimulation (Fig. 1D). Similarly, in two of seven cells tested at the far site, we could observe mGluR-mediated EPSC (Fig. $1 H$ ). To support the assumption that NBQX selectively acted on AMPA receptors, we recorded EPSCs from PCs in the near site during perfusion with the AMPA receptor agonist, kainic acid ( $1 \mu \mathrm{M})$, in the extracellular medium. We could not observe a response in any 
of the six tested cells during kainate perfusion (Fig. 1C), although this response was present at the far site (Fig. $1 G$ ).

An additional series of experiments has been addressed to find out the possible presence of residual quantal events after 2 $\mu \mathrm{mol}$ of NBQX administration. To this aim, we have recorded AMPA-mediated miniature events in the near and far sites of NBQX-treated animals. In untreated rats, we were able to record glutamatergic quantal events in the presence of $20 \mu \mathrm{M}$ bicuculline and $1 \mu \mathrm{M}$ TTX (Fig. $2 A$ ) in the perfusion medium. These miniature postsynaptic currents were completely blocked by adding $20 \mu \mathrm{M}$ NBQX (Fig. $2 B$ ). In three NBQX-infused animals, we recorded seven PCs at the near site. Here, we observed sustained spontaneous activity (Fig. 2C) that was completely abolished by bath application of bicuculline and TTX (Fig. 2D), thus revealing a complete absence of underlying glutamatergic activity. In contrast, in the far site, application of bicuculline and TTX disclosed miniature glutamatergic quantal events (Fig. 2G). These data show the presence of inhibitory GABAergic activity and confirm complete absence of miniature AMPA-mediated currents at the near site. In addition, we established the absence of evoked currents in response to PF stimulation (Fig. 2E). In three cells recorded at the far site, we could record PF-EPSCs (Fig. $2 F$ ) and detect miniature glutamatergic events in the presence of $20 \mu \mathrm{M}$ bicuculline and $1 \mu \mathrm{M}$ TTX (Fig. 2G), which were abolished by bath application of NBQX (Fig. $2 H$ ). These data all together demonstrate that a dose of $2 \mu \mathrm{mol}$ per week of NBQX is sufficient to induce a complete postsynaptic block of AMPA receptors without abolishing presynaptic release.

We followed a similar protocol of slice recording after MCPG administration. In three rats, we recorded nine PCs near the infusion site and another seven cells at the far site. In each cell, we first recorded the PF-EPSCs mediated by AMPA receptors. Such a current was present in all of the 16 recorded cells within a $2 \mathrm{~h}$ period, thus confirming that after MCPG infusion, the ionotropic glutamatergic transmission was still functional (Fig. $3 A, B)$. Then, we applied NBQX to block the AMPA-mediated current (Fig. $3 C, D$ ) and to be able to isolate the possible presence of mGluR-mediated EPSC. It has been shown that the mGluR postsynaptic response can be elicited by repetitive stimulation of PFs (Batchelor et al., 1994; Tempia et al., 1998). However, mGluRs are also present at the CF-PC synapse (Nusser et al., 1994; Petralia et al., 1998). Because their response requires the presence of glutamate uptake blockers to be clearly detectable (Dzubay and Otis, 2002; Zhu et al., 2005), we tested the effectiveness of the mGluRs block only through PF activation. In nine PCs located near the recording site, within the $2 \mathrm{~h}$ period, we found no trace of $\mathrm{mGluR}$-mediated response to PF stimulation (Fig. $3 E$ ). In contrast, such a response was present in five of seven cells recorded in the far site (Fig. $3 F$ ). These results show that our infusion protocol was effective to block AMPA- or mGluR-mediated synaptic transmission in PCs.
Vehicle

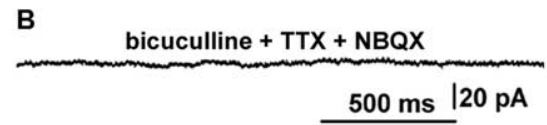

far site

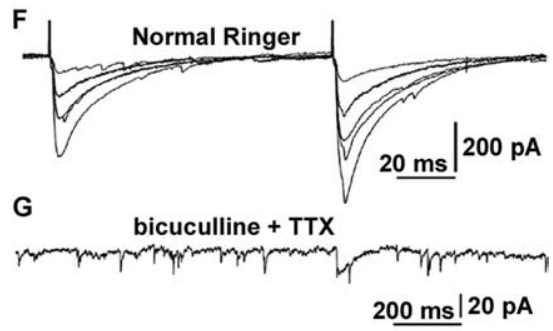

H bicuculline + TTX + NBQX

20 ms $\mid 200$ pA

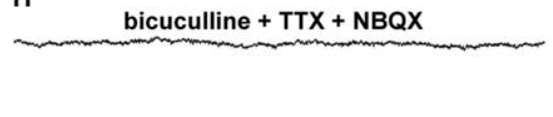

Figure 2. Recordings of AMPA-mediated miniature events in vehicle- and NBQX-treated cerebella. $A$, Glutamatergic miniature 作

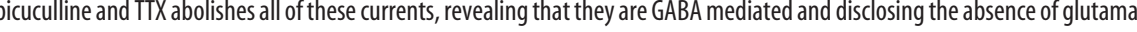
miniature AMPA-mediated glutamatergic activity $(\boldsymbol{G})$ that is abolished by addition of NBQX $(\boldsymbol{H})$.

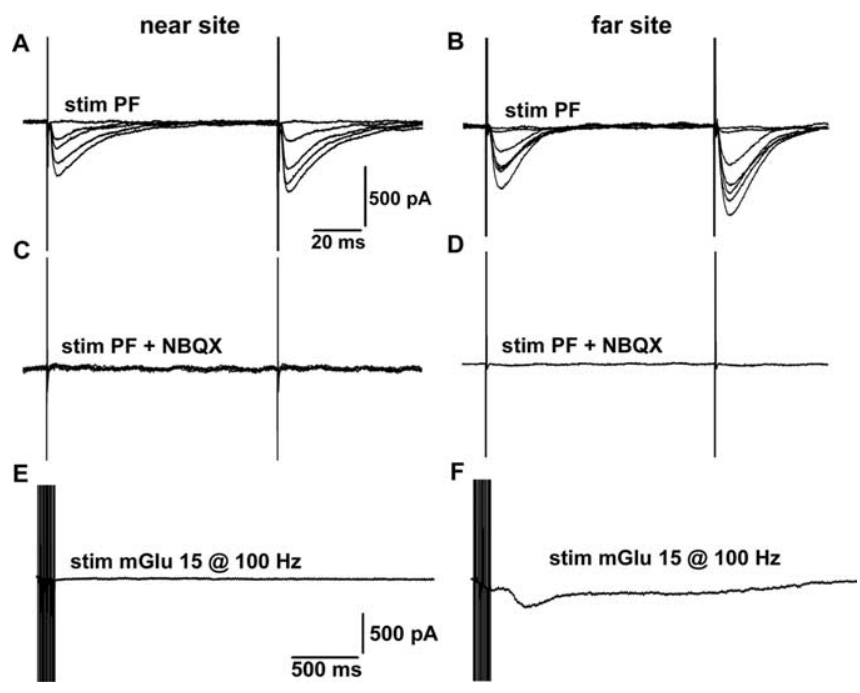

Figure 3. Electrophysiological recording in MCPG-treated cerebella. $\boldsymbol{A}-\boldsymbol{B}$, Assessment of the presence of PF responses in the near and far site of infusion. $\boldsymbol{C}-\boldsymbol{D}, \mathrm{NBQX}$ bath application abolishes these responses, demonstrating that they are AMPA mediated. $\boldsymbol{E}$, A train of stimuli in the PFs does not elicit mGluR-mediated EPSC at the near site of infusion, whereas these currents are present in the same slice at the far site $(\boldsymbol{F})$. Stim, Stimulation.

\section{Effects of glutamate receptor antagonists on PC dendritic spines}

First, we performed a quantitative confocal analysis of the density of spines emerging from the proximal dendritic compartment of the PCs, labeled with an anti-calbindin antibody, in vehicle-, MCPG-, and NBQX-treated cerebella for $7 \mathrm{~d}$ in vivo.

In control cerebella, the proximal dendrites appear relatively smooth compared with the distal dendrites that are entirely cov- 

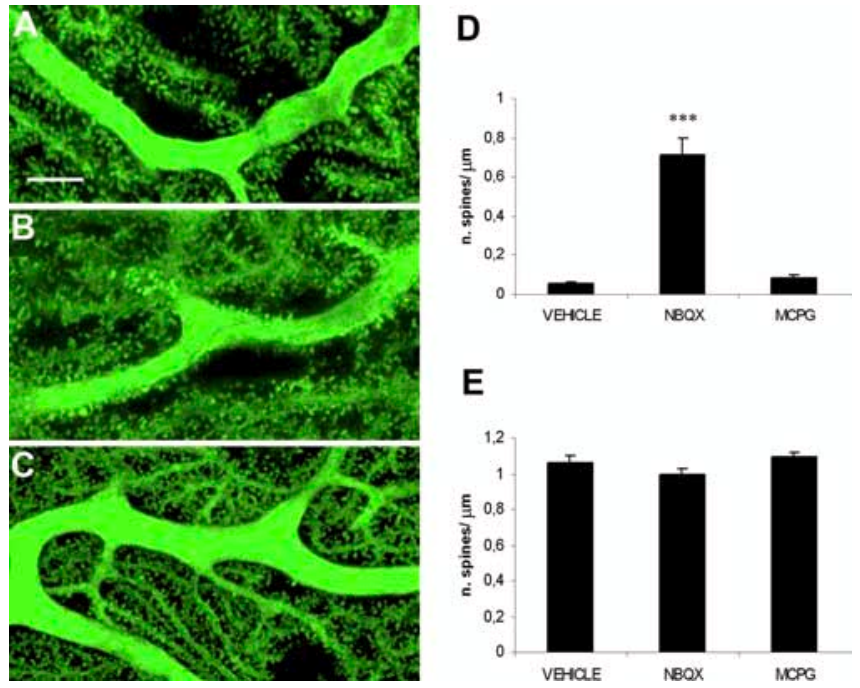

Figure 4. $\mathrm{PC}$ morphological modification after glutamate receptor blockade. $A, C$, The $\mathrm{PC}$ proximal dendritic segment is smooth in a vehicle-treated cerebellum $(\boldsymbol{A})$ and after MCPG infusion (C). $\boldsymbol{B}$, After NBQX treatment, the proximal dendrite is covered with spines. $\boldsymbol{D}, \boldsymbol{E}$, Histograms show the mean density of spines emerging from the proximal $(\boldsymbol{D})$ and from the distal (E) dendritic domain. The spine density after NBQX is significantly increased. ${ }^{* * *} p<0.001$. Error bars indicate SEM. Scale bar, $10 \mu \mathrm{m}$.

ered by numerous spines (Fig. 4A). The evaluation of spine density was assessed in samples of reconstructed PC proximal dendritic segments (see Materials and Methods) (Cesa et al., 2005). In the vehicle-treated cerebella, the mean spine density value, expressed as the number of spines per micrometer of dendrite length, was $0.056 \pm 0.01 \mathrm{SE}(n=48$ segments). The blockade of AMPA receptor by means of NBQX produced a clear-cut effect on the PC proximal dendrite (Fig. $4 B, D$ ): we observed an increase in spine density that reached a value of $0.716 \pm 0.08 \mathrm{SE}$ $(n=62 ; p<0.001)$. This structural modification was restricted to the site of infusion-adjacent lobules, consistent with previous results in which it has been reported that NBQX has a limited area of diffusion (Zhao and Davis, 2004). The mGluR antagonist MCPG produced a slight but not significant increase in spine density with a value of $0.086 \pm 0.01 \mathrm{SE}(n=45 ; p>0.05)$ (Fig. $4 C, D)$.

We also measured the spine density in the distal dendritic domain by counting the spines emerging from the dendrites. We did not find any significant differences with a value of $1.063 \pm$ 0.016 SE ( $n=36$ segments; total number of spines 228 ) in the vehicle-treated cerebella, and $1.100 \pm 0.009(n=50$, total number of spines, 217) and 1.086 $\pm 0.010 \mathrm{SE}(n=43$; total number of spines, 238) after NBQX and MCPG infusion, respectively ( $p>$ 0.05 ) (Fig. $4 E$ ). These values are smaller relative to the absolute number of spines per dendritic length (Harvey et al., 2005). However, this approach is valid for evaluation of the difference in density among the experimental groups.

Therefore, after NBQX infusion, in the presence of unaffected mGluRs, a large number of new spines appear in the proximal dendritic domain, whereas in the distal one, there are no significant changes. These results show that $\mathrm{CF}$ exerts its repressive action on PC spinogenesis through ionotropic AMPA/kainate receptors.

\section{CF modifications after glutamate receptor antagonist infusion}

Because the block of AMPA/kainate receptors induced the growth of a high number of spines in the PC proximal dendritic domain, we investigated whether these conditions also affected the presynaptic structure. We labeled cerebellar sections with an antibody raised against VGlut2 selectively expressed in the molecular layer of the adult cerebellum by the CF terminal varicosities (Fremeau et al., 2001; Miyazaki et al., 2003). First, in each experimental group, we calculated the number of the CF varicosities juxtaposed on segments of proximal dendrites identified in 15 randomly selected rectangular areas of molecular layer having the dimension of $80 \times 106 \mu \mathrm{m}$. The mean density values were expressed as the number of labeled varicosities per micrometer of dendrite length. In the vehicle-treated cerebella, we found an average of $0.3 \pm 0.01 \mathrm{SE}$ ( $n=35$ segments). The pharmacological treatment with MCPG and NBQX does not affect the CF varicosity density, the values being $0.31 \pm 0.02(n=29)$ and $0.33 \pm 0.02$ $\mathrm{SE}(n=40)$, respectively (Fig. $5 A)$. To confirm this result, we also measured the CF varicosity density, expressed as number of varicosities per square micrometer of molecular layer. We did not find any significant differences after receptor blockade, the values being $0.014 \pm 0.0007\left(n=15\right.$; total area, $\left.463,800 \mu \mathrm{m}^{2}\right), 0.012 \pm$ $0.0007\left(n=22\right.$; total area, 592,740 $\left.\mu \mathrm{m}^{2}\right)$, and $0.013 \pm 0.0007 \mathrm{SE}$ $\left(n=13\right.$; total area, 565,800 $\left.\mu \mathrm{m}^{2}\right)$, in the vehicle-, NBQX-, and MCPG-treated rats, respectively (Fig. 5B). Therefore, by blocking glutamate receptors, the CF terminal arbor does not present any modification in the number of varicosities. Also, the CF varicosity extension in the molecular layer did not show any alteration after the antagonist infusion: $84.9 \pm 3.0(n=16), 84.5 \pm 3.20$ $(n=22)$, and $86.40 \pm 2.60 \%(n=12)$ in the vehicle-, NBQX-, and MCPG-treated rats, respectively (Fig. 5C). These data show that after blockage of glutamate receptors, the total length of the CF terminal arbor and the density of its varicosity are unchanged. However, we noticed a morphological modification: large irregularly shaped boutons typical of the vehicle-treated cerebella (Fig. 5D) become shorter and round-shaped after NBQX infusion (Fig. 5E) and are not affected after MCPG treatment (Fig. $5 F$ ). We measured major axis, minor axis (in micrometers), and ratio (major/minor axis length) of randomly selected varicosities in the different experimental conditions. As shown in Table 1, the major axis length shows a significant reduction from a control value of $2.36 \pm 0.06 \mathrm{SE}(n=105)$ to a value of $1.56 \pm 0.03 \mathrm{SE}(n=$ 99) $(p<0.05)$ in the NBQX-treated cerebella, whereas the minor axis revealed no difference in values $(1.07 \pm 0.03$ vs $1.13 \pm 0.02$ $\mathrm{SE}$ ). The value of the ratio (major/minor axis length) showed a significant reduction after NBQX, indicating a structural modification of the varicosities from an elongated to a rounded shape. Thus, although after glutamate receptor blockade a change in the number of VGlut2-labeled CF varicosities did not occur, we observed a presynaptic structural plasticity dependent on activation of AMPA and kainate receptors.

\section{Ultrastructural CF varicosity and synaptic contact analysis}

We verified whether the CF varicosity morphological modification was characterized also by a reduction in the number of synaptic contacts on the PC. Using the electron microscope, we randomly selected CF varicosities in vehicle- and NBQX-treated cerebella. First, we measured major axis and minor axis length (in micrometers), ratio (major/minor axis length), area, and perimeter of the varicosities to support the confocal analysis results. Second, we quantified the number of spines contacting each varicosity (Table 2). We confirmed the significant reduction of the CF varicosity major axis length from a value of $2.36 \pm 0.15 \mathrm{SE}$ $(n=34)$ in the vehicle-treated cerebella to a value of $1.85 \pm 0.12$ $\mathrm{SE}(n=59)$ in the NBQX-infused rats $(p<0.01)$ (Fig. $5 G, H)$. The mean value of the ratio significantly changed similar to that 
of the perimeter $(p<0.05)$. Then, we quantified the number of spines contacting the varicosities in the two experimental conditions. We found significantly different mean values of $1.85 \pm 0.15$ and $1.19 \pm 0.12 \mathrm{SE}$ spines per CF terminal in the vehicle- and NBQX-treated animals, respectively $(p<0.01)$ (Fig. 5G,H). Therefore, in addition to a decreased size of presynaptic terminals, we also demonstrated a significant loss of synaptic contacts between CFs and the PCs.

\section{Ultrastructural analysis of the newly formed spine innervation after NBQX infusion}

In control conditions, all proximal spines are innervated by CF varicosities (Larramendi and Victor, 1967; Palay and ChanPalay, 1974) (Fig. 6C). Because NBQX induced the formation of spines in the PC proximal dendritic domain without affecting the distribution of the CF terminal arbor, we identified the nature of the presynaptic elements making contact with them according to specific morphological criteria. The excitatory boutons contain round vesicles and contact the PC dendrite on the spines forming asymmetric synapses; small clusters of vesicles abutting the synaptic contact characterize PF terminals, whereas CFs contain a high density of vesicles and some dense core vesicles uniformly distributed inside the boutons. GABAergic interneurons are characterized by round and oval or flattened vesicles and form symmetric-type synapses onto the smooth surface of the dendrite (Uchizono, 1967; Palay and Chan-Palay, 1974). We selected under the electron microscope those spines in continuity with a proximal dendritic profile. We analyzed 113 spines and 32 of them (28\%) were free of innervation (Fig. 6A). As shown in Figure 6B, of 81 innervated spines, $10(12.3 \%)$ had GABAergic terminals (Fig. 6E). These terminals have the characteristics to have a symmetric synapse onto the smooth dendritic surface in addition to the spine synapse. A second group of 63 spines (77.8\%) were in contact with profiles typical of PF boutons, characterized by small clusters of vesicles abutting the synaptic contact (Fig. $6 B, E$ ). The remaining eight spines $(9.9 \%)$ were contacted by CF varicosities (Fig. $6 B, D)$. This pattern of innervation is similar to that described after the block of the electrical activity: after TTX application, $9.8 \%$ of the proximal spines were innervated by GABAergic interneurons, $84.5 \%$ by PF boutons, and $5.7 \%$ by CF varicosities (Morando et al., 2001). Therefore, also in this condition, a large majority of the newly formed spines are innervated by PFs, whereas the remainders are innervated by GABAergic terminals or remain in contact with CFs.

\section{Discussion}

\section{Mechanisms of spinogenesis}

In the absence of electrical activity, the PC dendrite becomes a uniform territory with a high density of spines mainly innervated by the PFs. A similar picture is obtained when the CF terminal arbor degenerates after chemical or surgical lesions (Sotelo et al., 1975; Cesa et al., 2005). Spine density in the two territories returns to a normal level after electric block removal or if the de-
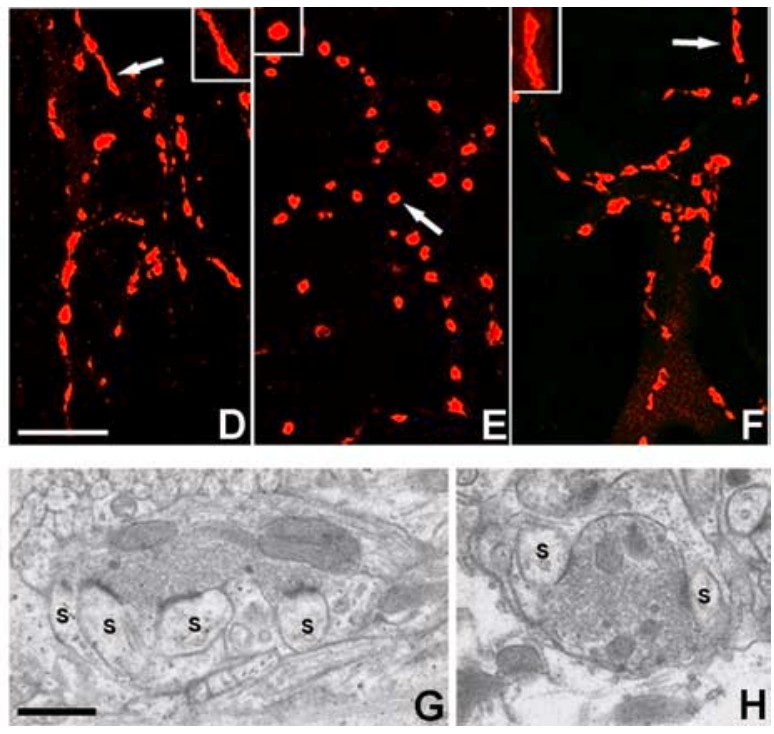

Figure 5. CF modifications after glutamate receptor antagonist infusion. $\boldsymbol{A}, \boldsymbol{B}$, The mean density values, expressed as the
number of labeled varicosities per micrometer of $P($ proximal dendrite length $(\boldsymbol{A})$ and per square micrometer of molecular layer $(\boldsymbol{B})$ are not affected by pharmacological treatment. $C$, Also, the extension of (F varicosities in the molecular layer does not show any alteration. Error bars indicate SEM. D-F, Long, irregularly shaped boutons of a vehicle-treated cerebellum (D), round-shaped ricosities after NBQX infusion $(\boldsymbol{E})$, and unaffected varicosities after $M C P G$ infusion $(\boldsymbol{F})$. Arrows indicate the varicosities shown at spines in a vehicle-treated cerebellum $(\boldsymbol{G})$, round-shaped varicosity after NBOX infusion $(\boldsymbol{H})$. Note the reduction of the number synaptic contacts with the spines in $\boldsymbol{H}$. S, Spine. Scale bars: (in $\boldsymbol{D}) \boldsymbol{D}-\boldsymbol{F}, 8 \mu \mathrm{m}$; (in $\boldsymbol{G}) \mathbf{G}, \boldsymbol{H}, 1 \mu \mathrm{m}$.

Table 1. Morphological analysis of CF varicosities in vehicle-treated cerebella (V) after NBQX $(N)$ and MCPG $(M)$ infusions

\begin{tabular}{llll}
\hline & $\mathrm{MA}(\mu \mathrm{m} \pm \mathrm{SE})$ & $\mathrm{ma}(\mu \mathrm{m} \pm \mathrm{SE})$ & $\mathrm{MA} / \mathrm{ma}(\mu \mathrm{m} \pm \mathrm{SE})$ \\
\hline $\mathrm{V}(n=105)$ & $2.36 \pm 0.06$ & $1.07 \pm 0.03$ & $2.34 \pm 0.08$ \\
$\mathrm{~N}(n=99)$ & $1.56 \pm 0.03$ & $1.13 \pm 0.02$ & $1.43 \pm 0.04$ \\
$\mathrm{M}(n=90)$ & $2.19 \pm 0.06$ & $0.95 \pm 0.03$ & $2.47 \pm 0.09$ \\
\hline
\end{tabular}

Mean values of major axis length (MA), minor axis length ( $\mathrm{ma})$, and ratio (MA/ma).

nervated PC is reinnervated by the collateral sprouting of surviving olivary neurons (Rossi et al., 1991a,b). These findings led to the hypothesis that (1) an activity-independent, intrinsic mechanism (Sotelo, 1978) promotes spine growth over the whole dendritic territory, whereas (2) an activity-dependent spine-pruning action is exerted by the CF at the proximal dendrites around its synapses as a kind of lateral inhibition (Cesa and Strata, 2005).

\section{Intrinsic mechanisms}

Different models of spinogenesis have been proposed. In the first model, dendritic filopodia grow toward nearby axons and become spines (Nimchinsky et al., 2002). In a second model, presynaptic molecules in axons might induce the expansion of the head of a filopodium and make it become a spine. Axons might even be able to induce the extension of dendritic spines directly from dendritic shafts (Nimchinsky et al., 2002; Penzes et al., 2003). The first model applies better to the PC spines, which are able to grow even in the absence of presynaptic terminals (Yuste and Bonhoeffer, 2004). In weaver mutant mice, PCs lack their main excitatory granular cell afferents and still bear spines (Hirano et al., 1977). After elimination of granule cells with methylazoxymethanol acetate (Takács et al., 1997) or with X-irradiations (Baloyannis and Kim, 1979), free PC spines develop and present postsynaptic densities.

Such a view seems in contrast with the notion that spines in PC dendrites grow under the influence of PFs, as suggested by in vitro 
Table 2. Ultrastructural CF varicosity morphological analysis in vehicle- and NBQX-treated cerebella

\begin{tabular}{|c|c|c|c|c|c|c|}
\hline & $\mathrm{MA}(\mu \mathrm{m} \pm \mathrm{SE})$ & $\mathrm{ma}(\mu \mathrm{m} \pm \mathrm{SE})$ & $\mathrm{MA} / \mathrm{ma}(\mu \mathrm{m} \pm \mathrm{SE})$ & Area $\left(\mu \mathrm{m}^{2} \pm \mathrm{SE}\right)$ & $\begin{array}{l}\text { Perimeter } \\
(\mu \mathrm{m} \pm \mathrm{SE})\end{array}$ & $\begin{array}{l}\text { Spines per varicosity } \\
(n \pm \text { SE) }\end{array}$ \\
\hline \multicolumn{7}{|l|}{ Vehicle } \\
\hline$(n=34)$ & $2.36 \pm 0.15$ & $0.95 \pm 0.18$ & $3.88 \pm 0.56$ & $1.51 \pm 0.10$ & $6.55 \pm 0.36$ & $1.85 \pm 1.13$ \\
\hline \multicolumn{7}{|l|}{ NBQX } \\
\hline$(n=59)$ & $1.85 \pm 0.12$ & $0.93 \pm 0.12$ & $2.34 \pm 0.35$ & $1.46 \pm 0.10$ & $5.32 \pm 0.28$ & $1.19 \pm 0.92$ \\
\hline
\end{tabular}

Mean values of major axis length (MA), minor axis length (ma), ratio (MA/ma), area, perimeter, and density of spines contacting the varicosities.
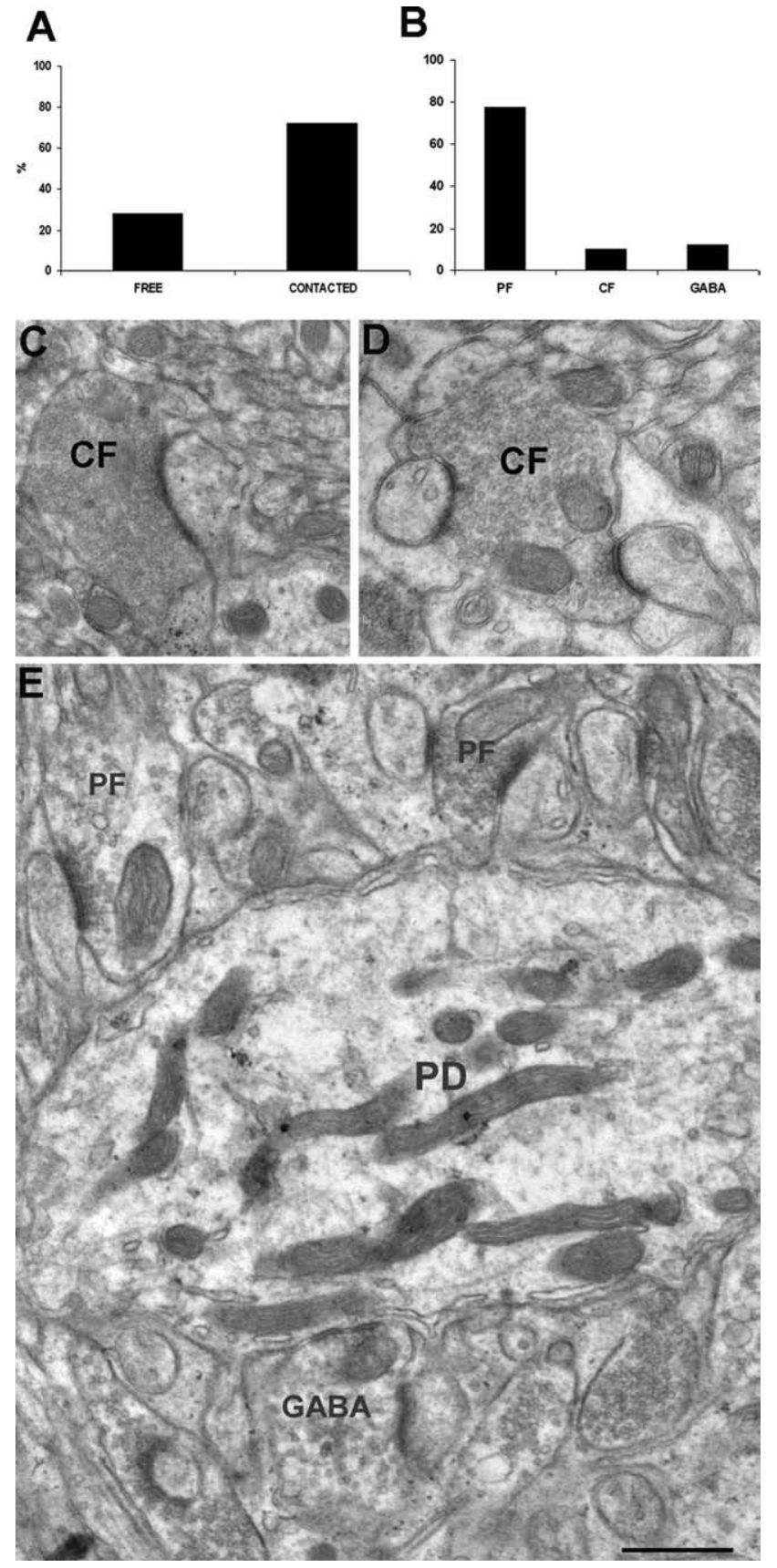

Figure 6. Ultrastructural analysis of the newly formed spine innervation after NBQX infusion. $A$, Percentage of distribution of free and contacted spines. Percentage of distribution of the type of innervation of the proximal dendritic spines after NBQX treatment. $B$, The three columns represent the complement innervated by PFs, CFs, and GABAergic terminals. C, In control conditions, proximal dendritic spines are innervated by $C F$ varicosities. $\boldsymbol{D}$, A spine emerging from a $P C$ proximal dendrite innervated by a $C F$ varicosity in the NBQX-treated cerebellum. $E$, Spines emerging from a proximal dendrite (PD) contacted by PFs and by a GABAergic terminal. Scale bar: (in $\boldsymbol{E}) \mathbf{C}-\boldsymbol{E}, 1 \mu \mathrm{m}$. experiments (Baptista et al., 1994). Studies in which PCs are cultured alone or with granule neurons showed that PC survival and differentiation require a balance between the neurotrophin and neurotransmitter signaling and that BDNF treatment increases the PC spine density (Morrison and Mason, 1998; Shimada et al., 1998).

The discrepancy might be because of the different experimental conditions. However, the presence of in vivo environmental factors that could mediate an extrinsic activity-independent mechanism in PC spinogenesis cannot be excluded. Possible mechanisms might involve diffusible substances as BDNF, but also ligand molecules in the presynaptic membrane like ephrinB binding to EphB receptors located in the postsynaptic membrane (Aoto and Chen, 2006). The latter mechanism is unlikely to be operative in spines that may exist without a presynaptic partner. Among the diffusible substances, quantal glutamate release maintains hippocampal spines (McKinney et al., 1999). Because PC spinogenesis in proximal dendrites occurs under TTX treatment where quantal release is not abolished, we tested whether glutamate release from neuronal and non-neuronal elements like glia (Mazzanti et al., 2001; Fellin et al., 2005) is essential for spine growth. To face this issue, we infused a specific glutamate receptor antagonist in vivo to block AMPA/kainate and mGluR receptors.

To prove the complete block of the receptors, we showed the absence of any detectable spontaneous quantal event and of evoked EPSC to PF and CF stimulation near the infusion site. The dose of NBQX necessary to obtain such a block was $2 \mu \mathrm{mol}$ in 100 $\mu \mathrm{l}$ of physiological solution during a period of $7 \mathrm{~d}$, whereas with a dose of 1 and $1.5 \mu \mathrm{mol}$, the block was incomplete. Therefore, the effects of $2 \mu \mathrm{mol}$ are likely similar to those obtained on acute slices with a concentration of $10-20 \mu \mathrm{M}$ currently used in the literature (Batchelor et al., 1997; Scelfo et al., 2003). However, a prolonged administration of NBQX might exert additional changes, which are absent in acute conditions. Kakizawa et al. (2005) described the effects of such a prolonged administration. These authors applied the blocker on the surface of the cerebellum by an Elvax implant, and they found that after the washout of the slices, the strength of the postsynaptic current was reduced because of a decrease of the number of the release sites, everything else being unchanged. In our experiments, in which NBQX was administered intraparenchymally at a concentration to completely abolish the postsynaptic response, we were unable to demonstrate such a recovery. However, we tested whether the lack of AMPA-mediated response was because of a complete postsynaptic block or to the absence of presynaptic release.

To verify that presynaptic release still occurs in these conditions, we assessed the presence of mGluR-mediated responses with a short high-frequency train of pulses to the PFs. In addition, we proved that postsynaptic receptors were indeed blocked, because in NBQX-infused animals, the perfusion with kainate, an AMPA receptor agonist, did not elicit AMPA-mediated response in the near site, but it did in the far site. Similarly, with MCPG, we 
assessed the absence of mGluR-mediated responses in the near site of infusion where PF-mediated EPSCs were still present, thus demonstrating the postsynaptic action of the blocker.

Concerning spinogenesis, we found that after a complete block of AMPA-mediated EPSCs and quantal events, there was a remarkable increase of spines in the PC proximal dendrites. The way PCs produce spines is at variance with that of hippocampal neurons. In fact, in the latter structure, a loss of spines has been described as the result of presynaptic deafferentation and after block of AMPA-mediated activity with botulinum toxin or NBQX (McKinney et al., 1999).

\section{Spine pruning}

Our experiments also show that an increase in spine density occurs in the PC proximal dendrites of the NBQX-treated rats, whereas MCPG is ineffective to induce spinogenesis. In contrast, in the distal dendrites, spine density was not affected by infusion of both blockers. Therefore, we can conclude that the active CFs exert repressive action on the dendritic domain surrounding their synapses through ionotropic AMPA/kainate receptors.

\section{Presynaptic structural plasticity}

In the rat, each CF terminal arbor has nearly 300 varicosities (Rossi et al., 1993) and each of them makes contact with a cluster of a few spines (Palay and Chan-Palay, 1974). We showed that after NBQX treatment, both number and density of these presynaptic structures are unmodified, but a change in their shape occurs with a significant decrease in the number of contacted spines. In normal conditions, the varicosity has an elongated and lobate profile, likely because of its adaptation to cover the spine clusters. The more rounded shape acquired after block of AMPA receptors likely reflects the reduced number of spines that are in contact with each varicosity. This decrease is consistent with the diminution of the number of release sites after chronic NBQX treatment, demonstrated at the electrophysiological level by Kakizawa et al. (2005). At a variance with our results, Kakizawa et al. (2005) observed that this reduction of release sites is accompanied by a retraction of CF terminal arbor. Furthermore, Kakizawa et al. (2005) did not find a hyperspiny transformation, not even in the part of the PC dendrite that lost the CF innervation. Such a discrepancy might be because of some basic differences in the experimental conditions: we used osmotic minipumps that constantly delivered the antagonists during a $7 \mathrm{~d}$ period through a cannula directly inserted inside the cerebellar parenchyma, whereas the Elvax implant used by Kakizawa et al. (2005) was placed on the surface of the cerebellum. Our protocol allows determining precisely the amount of blocker delivered in a week, and this is not possible with the Elvax technique. In addition, in our conditions, we could observe a complete block of PF- and CF-EPSCs and also of spontaneous quantal events even in the acute slices up to $2 \mathrm{~h}$ after the preparation.

The present experiments support the hypothesis that PCs have the intrinsic ability to promote spines over the entire dendritic territory independently from glutamate influence. Each spine is then innervated by a PF. The active CF has a sculpturing effect on the uniform distribution of the dendritic spines. In fact, it acquires and maintains its territory by increasing the number of synaptic contacts under each varicosity and by repressing spinogenesis in the nearby-located dendritic territory, thus displacing the PF input. The tendency for the CF synapses to become weaker after TTX or NBQX treatment have been explained as a retrograde effect (Kakizawa et al., 2005), because such weakening occurs in mutant mice deficient in $\mathrm{P} / \mathrm{Q}$-type $\mathrm{Ca}^{2+}$ channel $\alpha 1 \mathrm{~A}$ subunit with a consequent deficiency in $\mathrm{Ca}^{2+}$ level in PCs (Miyazaki et al., 2004). However, the hypothesis that glutamate receptor blockage prevents the release of an intracellular messenger involved in the maintenance of the presynaptic varicosity normal features cannot be excluded. In fact, in hippocampal slice cultures, it has been shown that NMDA receptor activation and subsequent release of nitric oxide stimulates a presynaptic morphological plasticity that regulates synaptogenesis (Nikonenko et al., 2003). Finally, we cannot rule out the possibility that the reduced number of spines in each CF varicosity observed under NBQX administration may be because of a competitive mechanism with the newly formed spines, innervated by PFs, in the proximal dendritic domain.

\section{References}

Aoto J, Chen L (2007) Bidirectional ephrin/Eph signaling in synaptic functions. Brain Res, in press.

Baloyannis SJ, Kim SU (1979) Experimental modification of cerebellar development in tissue culture: $\mathrm{X}$-irradiation induces granular degeneration and unattached Purkinje cell dendritic spines. Neurosci Lett 12:283-288.

Baptista CA, Hatten ME, Blazeski R, Mason CA (1994) Cell-cell interactions influence survival and differentiation of purified Purkinje cells in vitro. Neuron 12:243-260.

Batchelor AM, Madge DJ, Garthwaite J (1994) Synaptic activation of metabotropic glutamate receptors in the parallel fibre-Purkinje cell pathway in rat cerebellar slices. Neuroscience 63:911-915.

Batchelor AM, Knöpfel T, Gasparini F, Garthwaite J (1997) Pharmacological characterization of synaptic transmission through mGluRs in rat cerebellar slices. Neuropharmacology 36:401-403.

Bravin M, Morando L, Vercelli A, Rossi F, Strata P (1999) Control of spine formation by electrical activity in the adult cerebellum. Proc Natl Acad Sci USA 96:1704-1709.

Cesa R, Strata P (2005) Axonal and synaptic remodeling in the mature cerebellar cortex. Prog Brain Res 148:45-56.

Cesa R, Morando L, Strata P (2005) Purkinje cell spinogenesis during architectural rewiring in the mature cerebellum. Eur J Neurosci 22:579-586.

Dzubay JA, Otis TS (2002) Climbing fiber activation of metabotropic glutamate receptors on cerebellar purkinje neurons. Neuron 36:1159-1167.

Edwards FA, Konnerth A, Sakmann B, Takahashi T (1989) A thin slice preparation for patch clamp recordings from neurones of the mammalian central nervous system. Pflügers Arch 414:600-612.

Engert F, Bonhoeffer T (1999) Dendritic spine changes associated with hippocampal long-term synaptic plasticity. Nature 399:66-70.

Fellin T, Pascual O, Haydon PG (2005) Astrocytes coordinate synaptic network: balanced excitation and inhibition. Physiology 21:208-215.

Fremeau Jr RT, Troyer MD, Pahner I, Nygaard GO, Tran CH, Reimer RJ, Bellocchio EE, Fortin D, Storm-Mathisen J, Edwards RH (2001) The expression of vesicular glutamate transporters defines two classes of excitatory synapse. Neuron 31:247-260.

Harvey RJ, Morando L, Rasetti R, Strata P (2005) Spontaneous electrical activity and dendritic spine size in mature cerebellar Purkinje cells. Eur J Neurosci 21:1777-1784.

Hirano A, Dembitzer HM, Yoon CH (1977) Development of Purkinje cell somatic spines in the weaver mouse. Acta Neuropathol 40:85-90.

Ichikawa R, Miyazaki T, Kano M, Hashikawa T, Tatsumi H, Sakimura K, Mishina M, Inoue Y, Watanabe M (2002) Distal extension of climbing fiber territory and multiple innervation caused by aberrant wiring to adjacent spiny branchlets in cerebellar Purkinje cells lacking glutamate receptor $\delta 2$. J Neurosci 22:8487-8503.

Kakizawa S, Miyazaki T, Yanagihara D, Iino M, Watanabe M, Kano M (2005) Maintenance of presynaptic function by AMPA receptor-mediated excitatory postsynaptic activity in adult brain. Proc Natl Acad Sci USA 102:19180-19185.

Knott GW, Holtmaat A, Wilbrecht L, Welker E, Svoboda K (2006) Spine growth precedes synapse formation in the adult neocortex in vivo. Nat Neurosci 9:1117-1124.

Konnerth A, Llano I, Armstrong CM (1990) Synaptic currents in cerebellar Purkinje cells. Proc Natl Acad Sci USA 87:2662-2665.

Lambolez B, Audinat E, Bochet P, Crepel F, Rossier J (1992) AMPA receptor subunits expressed by single Purkinje cells. Neuron 9:247-258. 
Larramendi LMH, Victor T (1967) Synapses on the Purkinje cell spines in the mouse. An electronmicroscopic study. Brain Res 5:15-30.

Llinàs R, Sugimori M (1980) Electrophysiological properties of in vitro Purkinje cell dendrites in mammalian cerebellar slices. J Physiol (Lond) 305:171-195.

Mazzanti M, Sul JY, Haydon PG (2001) Glutamate on demand: astrocytes as a ready source. Neuroscientist 7:396-405.

McKinney RA, Capogna M, Durr R, Gähwiler BH, Thompson SM (1999) Miniature synaptic events maintain dendritic spines via AMPA receptor activation. Nat Neurosci 2:44-49.

Miyazaki T, Fukaya M, Shimizu H, Watanabe M (2003) Subtype switching of vesicular glutamate transporters at parallel fibre-Purkinje cell synapses in developing mouse cerebellum. Eur J Neurosci 17:2563-2572.

Miyazaki T, Hashimoto K, Shin HS, Kano M, Watanabe M (2004) P/Q-type $\mathrm{Ca}^{2+}$ channel alphalA regulates synaptic competition on developing cerebellar Purkinje cells. J Neurosci 24:1734-1743.

Morando L, Cesa R, Rasetti R, Harvey R, Strata P (2001) Role of glutamate $\delta$ - 2 receptors in activity-dependent competition between heterologous afferent fibers. Proc Natl Acad Sci USA 98:9954-9959.

Morrison ME, Mason CA (1998) Granule neuron regulation of Purkinje cell development: striking a balance between neurotrophin and glutamate signaling. J Neurosci 18:3563-3573.

Nikonenko I, Jourdain P, Muller D (2003) Presynaptic remodeling contributes to activity-dependent synaptogenesis. J Neurosci 23:8498-8505.

Nimchinsky EA, Sabatini BL, Svoboda K (2002) Structure and function of dendritic spines. Annu Rev Physiol 64:313-353.

Nusser Z, Mulvihill E, Streit P, Somogyi P (1994) Subsynaptic segregation of metabotropic and ionotropic glutamate receptors as revealed by immunogold localization. Neuroscience 61:421-427.

Palay SL, Chan-Palay V (1974) Cerebellar cortex: cytology and organization. Berlin: Springer.

Penzes P, Beeser A, Chernoff J, Schiller MR, Eipper BA, Mains RE, Huganir RL (2003) Rapid induction of dendritic spine morphogenesis by transsynaptic ephrinB-EphB receptor activation of the Rho-GEF kalirin. Neuron 37:263-274.

Perkel DJ, Hestrin S, Sah P, Nicoll RA (1990) Excitatory synaptic currents in Purkinje cells. Proc Biol Sci 241:116-121.

Petralia RS, Wenthold RJ (1992) Light and electron immunocytochemical localization of AMPA-selective glutamate receptors in the rat brain. J Comp Neurol 318:329-354.

Petralia RS, Zhao HM, Wang YX, Wenthold RJ (1998) Variations in the tangential distribution of postsynaptic glutamate receptors in Purkinje cell parallel and climbing fiber synapses during development. Neuropharmacology 37:1321-1334.

Rossi F, Wiklund L, van der Want JJ, Strata P (1991a) Reinnervation of cerebellar Purkinje cells by climbing fibres surviving a subtotal lesion of the inferior olive in the adult rat. I. Development of new collateral branches and terminal plexuses. J Comp Neurol 308:513-535.

Rossi F, Wiklund L, van der Want JJ, Strata P (1991b) Reinnervation of cerebellar Purkinje cells by climbing fibres surviving a subtotal lesion of the inferior olive in the adult rat. II. Synaptic organization on reinnervated Purkinje cells. J Comp Neurol 308:536-554.

Rossi F, Borsello T, Vaudano E, Strata P (1993) Regressive modifications of climbing fibres following Purkinje cell degeneration in the cerebellar cortex of the adult rat. Neuroscience 53:759-778.

Scelfo B, Strata P, Knöpfel T (2003) Sodium imaging of climbing fiber innervation fields in developing mouse Purkinje cells. J Neurophysiol 89:2555-2563.

Shimada A, Mason CA, Morrison ME (1998) TrkB signaling modulates spine density and morphology independent of dendrite structure in cultured neonatal Purkinje cells. J Neurosci 18:8559-8570.

Sotelo C (1978) Purkinje cell ontogeny: formation and maintenance of spines. Prog Brain Res 48:149-170.

Sotelo C, Hillman DE, Zamora AJ, Llinás R (1975) Climbing fiber deafferentation: its action on Purkinje cell dendritic spines. Brain Res 98:574-581.

Takács J, Gombos G, Gorcs T, Becker T, de Barry J, Hámori J (1997) Distribution of metabotropic glutamate receptor type 1a in Purkinje cell dendritic spines is independent of the presence of presynaptic parallel fibers. J Neurosci Res 50:433-442.

Tempia F, Miniaci MC, Anchisi D, Strata P (1998) Postsynaptic current mediated by metabotropic glutamate receptors in cerebellar Purkinje cells. J Neurophysiol 80:520-528.

Uchizono K (1967) Synaptic organization of the Purkinje cells in the cerebellum of the cat. Exp Brain Res 4:97-113.

Yuste R, Bonhoeffer T (2004) Genesis of dendritic spines: insights from ultrastructural and imaging studies. Nat Rev Neurosci 5:24-34.

Zhao Z, Davis M (2004) Fear-potentiated startle in rats is mediated by neurons in the deep layers of the superior colliculus/deep mesencephalic nucleus of the rostral midbrain through the glutamate non-NMDA receptors. J Neurosci 24:10326-10334.

Zhu L, Strata P, Andjus PR (2005) Pharmacology of the metabotropic glutamate receptor mediated current at the climbing fiber to Purkinje cell synapse. Prog Brain Res 148:299-306. 